\title{
Diabetic Holiday Foot: Cautionary Tales of Sun, Sandals, Risk
}

\section{J A O'Hare ${ }^{1}$, F Nolan¹, E Kavanagh², P Burke², E Carrig ${ }^{3}$}

Departments of Endocrinology ${ }^{1}$, Department Vascular Surgery ${ }^{2}$, Podiatry Unit ${ }^{3}$

University Hospital Limerick, Dooradoyle, and University of Limerick, Graduate Entry Espidéil OL

Medical School, Limerick, Ireland

\section{Introduction}

Polyneuropathy is the most prevalent complication of diabetes and is often present at the diagnosis of type 2 diabetes. It is a major risk for injury to the feet. This case series illustrates the hazards to diabetic patients who may reduce their guard, alter their footwear and participate in unaccustomed walking on uneven surfaces on holiday in warm climates.

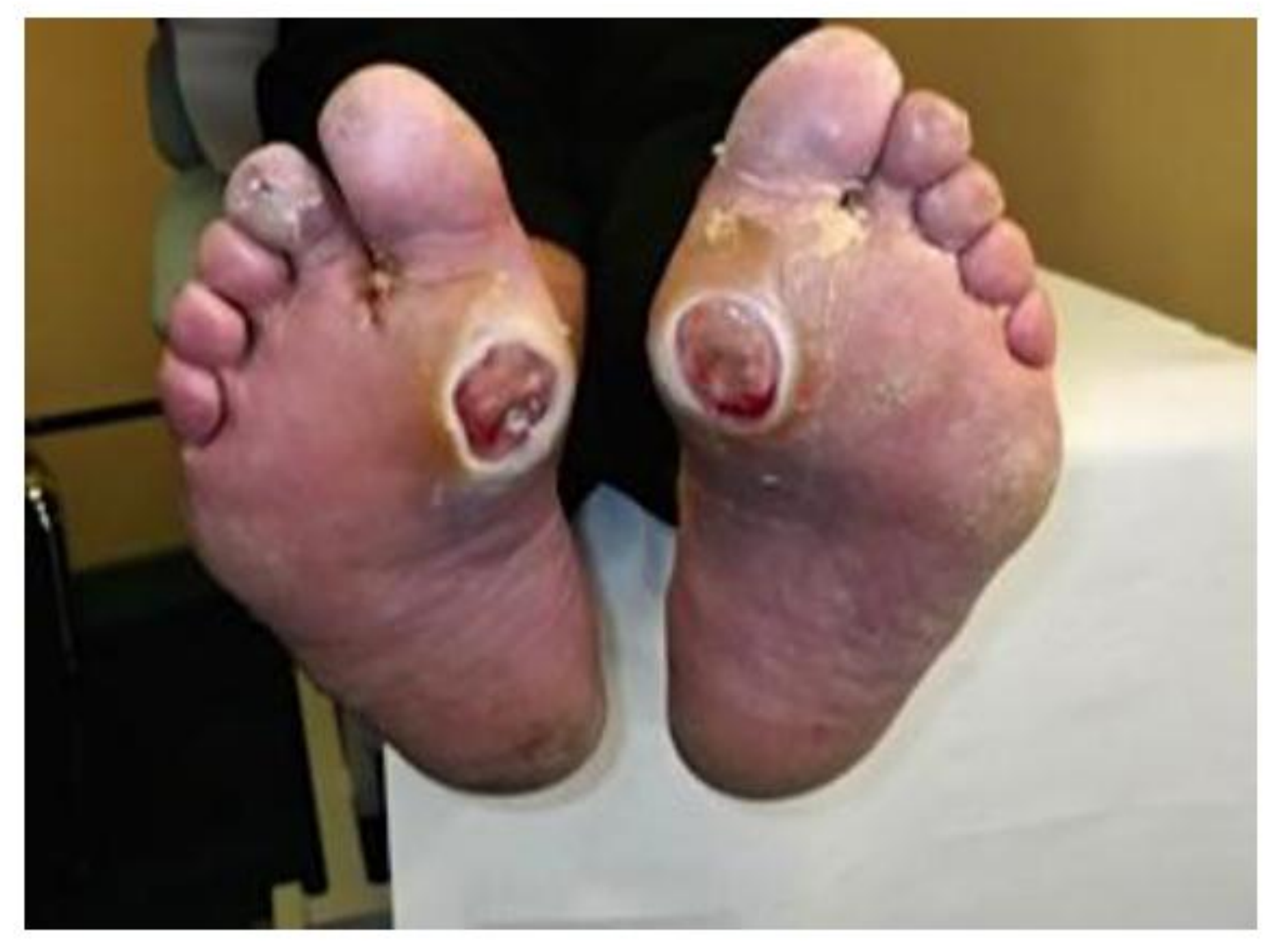

Patient "a"

Barefoot walking on sand and promenade, Spain $\times 2$ weeks metatarsal ulcers.

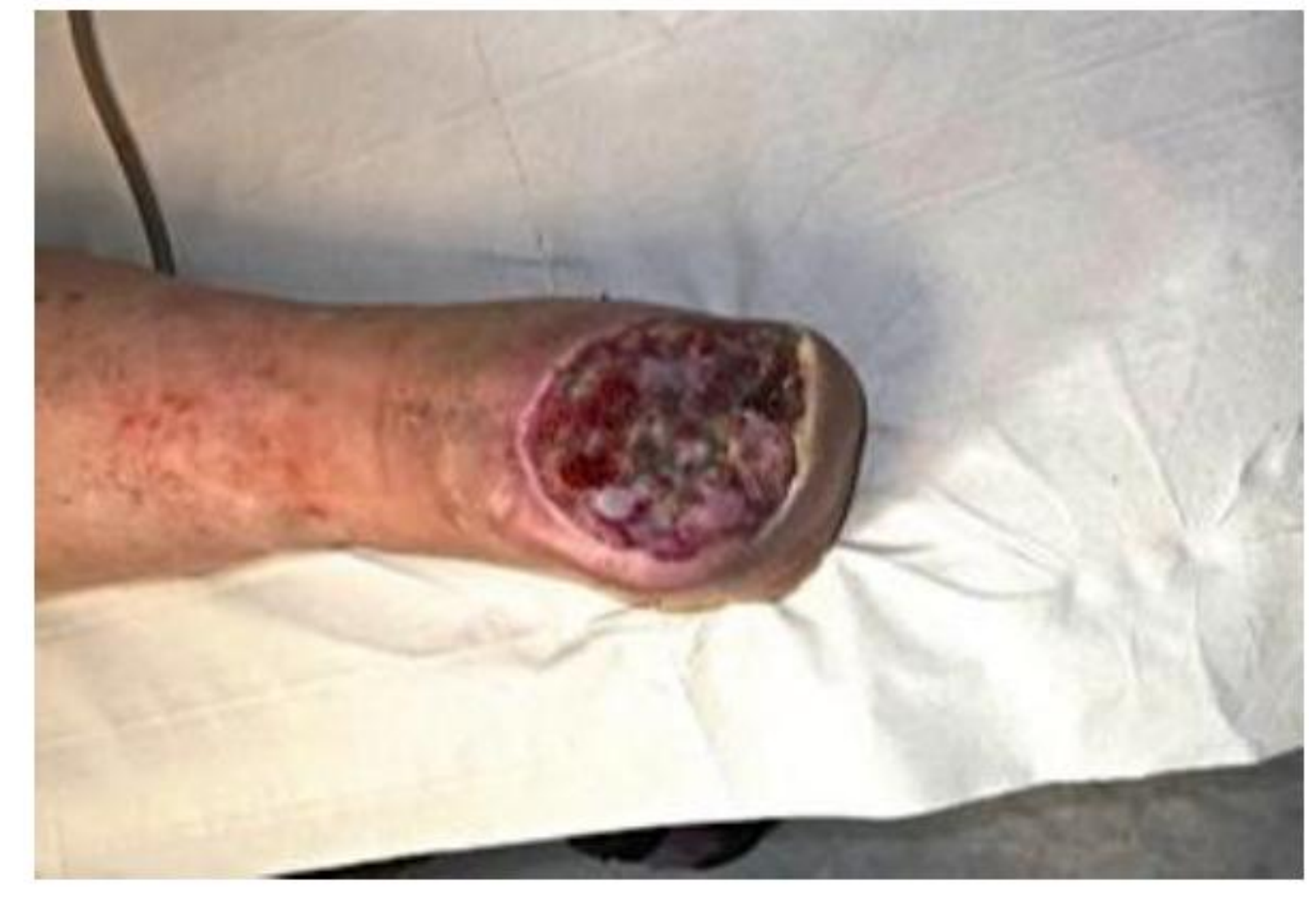

Patient " $b$ " Golf holiday in Spain, new golf shoes, pressure necrosis: eventual forefoot amputation

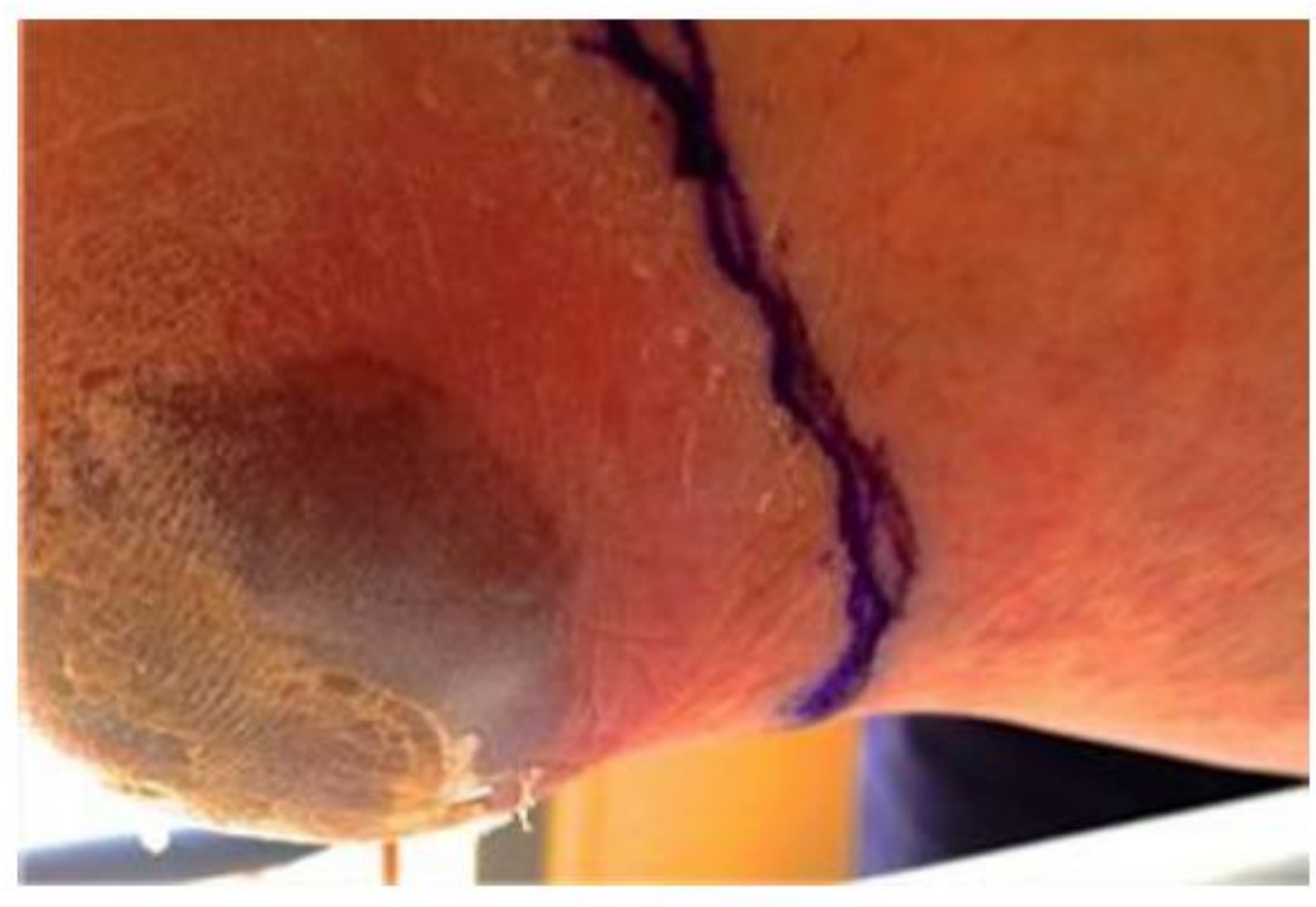

\section{Patient " $\mathrm{f}$ "}

Walking holiday in Croatia, cobbled surfaces, new sandals necrotic heel ulcer.

\begin{tabular}{|c|c|c|c|c|c|c|c|}
\hline & a & b & C & d & e & f & g \\
\hline $\begin{array}{l}\text { Age } \\
\text { (All male) }\end{array}$ & 57 & 62 & 58 & 68 & 59 & 58 & 59 \\
\hline DM Type & 2 & 2 & 2 & 2 & 1 & 2 & 2 \\
\hline Duration years & 15 & 19 & 5 & 12 & 30 & 15 & 1 \\
\hline Hba1c \% & 9.6 & 10.6 & 6.8 & 10 & 9.6 & 7.9 & 7.1 \\
\hline Smoker & no & no & no & no & yes & no & no \\
\hline Charcot Joint & no & no & yes & no & no & yes & no \\
\hline $\begin{array}{l}\text { Macrovascular } \\
\text { Disease }\end{array}$ & no & no & no & yes & yes & no & no \\
\hline Footwear & barefoot & Golf shoe & sandal & sandal & sandal & $\begin{array}{l}\text { Ortho. } \\
\text { boot }\end{array}$ & sandal \\
\hline Lesion & ulcer & necrosis & ulcer & $\begin{array}{l}\text { macera } \\
\text { tion }\end{array}$ & ulcer & blister & ulcer \\
\hline Location & planter & planter & Lateral & toes & planter & Heel & planter \\
\hline Amputation & no & forefoot & no & no & Part foot & no & no \\
\hline Healing weeks & 40 & 15 & 8 & 10 & 26 & 4 & 4 \\
\hline
\end{tabular}

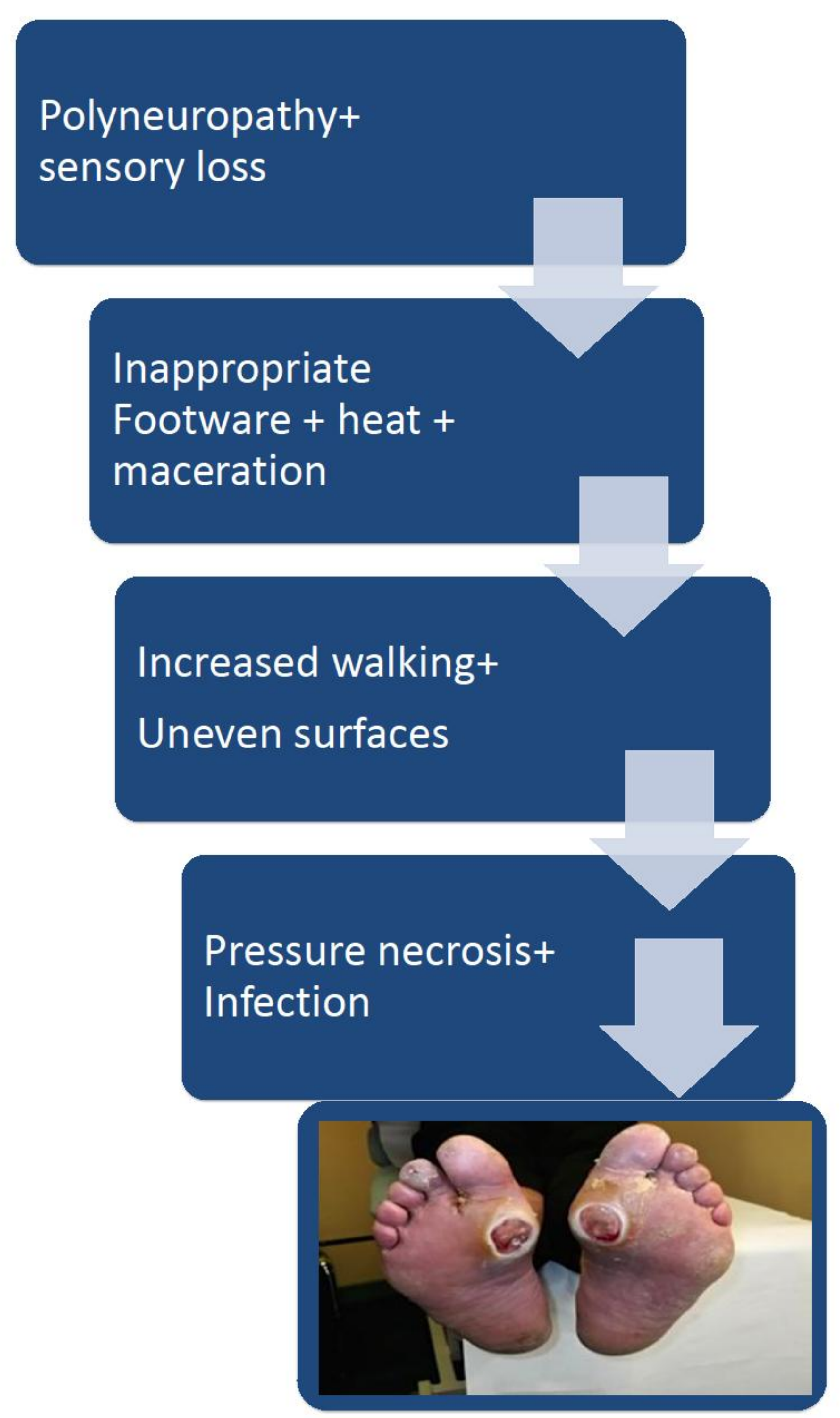

\section{Diabetic patients, especially males, should be warned of the risk to their feet}

